\title{
Ultrassom terapêutico associado a óleos essenciais de copaíba e melaleuca na cicatrização de lesões de pele
}

\section{Therapeutic ultrasound associated with essential oils of copaiba and tea tree for healing skin lesions}

\section{Ultrasonido terapéutico asociado con los aceites esenciales de copaíba y Melaleuca para curar lesiones cutáneas}

Darrielle Gomes Alves Mororó ${ }^{1}$, Maria Girlane Sousa Albuquerque Brandão ", , Vanessa Aguiar Ponte ${ }^{2}$, Alan Sidney Jacinto da Silva ${ }^{1}$, Francisco Walyson da Silva Batista ${ }^{1}$, Vivian Saraiva Veras ${ }^{1}$, Thiago Moura de Araújo ${ }^{1}$

ORCID IDS

Mororó DGA (D) https://orcid.org/0000-0002-9665-9007

Brandão MGSA (D) https://orcid.org/0000-0002-9925-4750

Ponte VA (D) https://orcid.org/0000-0002-2932-5509

Silva ASJ (D) https://orcid.org/0000-0003-2799-4992

Batista FWS (D) https://orcid.org/0000-0002-7238-6323

Veras VS (D) https://orcid.org/0000-0003-3267-3712

Araújo TM (D) https://orcid.org/0000-0002-8410-0337

\section{COMO CITAR}

Mororó DGA; Brandão MGSA; Ponte VA; Silva ASj; Batista FWS; Veras VS; Araújo TM. Ultrassom terapêutico associado a óleos essenciais de copaíba e melaleuca na cicatrização de lesões de pele. ESTIMA, Braz. J. Enterostomal Ther., 2020, 18: e3320. https://doi.org/10.30886/ estima.v18.942_PT

\section{RESUMO}

Objetivo: avaliar a eficácia do ultrassom de baixa frequência associado ao gel de óleos de copaíba e melaleuca na cicatrização de lesões de pele. Método: estudo quase-experimental com pré e pós-testes em grupo único, realizado em hospital público no Ceará, Brasil, no período de março a setembro de 2017, com 14 participantes que receberam 10 sessões de tratamento. Resultados: houve redução média de mais de $20 \mathrm{~cm}^{2}$ da $1^{a}$ para $5^{a}$ aplicação. A redução no tamanho inicial para a $10^{a}$ sessão foi superior a 50\%. Houve significância estatística ( $p=0,0043$ ) entre a avaliação inicial e a $10^{a}$ avaliação da terapia, com redução média de 37 cm². Houve redução na intensidade da dor relatada pelos participantes e 62,5\% estavam livres da dor após aplicação da terapia. Conclusão: o uso do gel com óleo de copaíba e melaleuca aponta para uma alternativa de cobertura associada a bioestimuladores como o ultrassom. A composição do óleo em gel auxiliou na aplicação e na redução de maceração do bordo das feridas bem como o controle do exsudato da lesão.

DESCRITORES: Cicatrização; Terapia por ultrassom; Óleos voláteis. Estomaterapia.

1. Universidade da Integração Internacional da Lusofonia Afro-Brasileira - Instituto de Ciências da Saúde - Redenção (CE), Brasil.

2. Universidade Federal do Ceará - Departamento de Enfermagem - Fortaleza (CE), Brasil.

*Autora correspondente: girlane.albuquerque@yahoo.com.br

Recebido: Set. 12, 2020 | Aceito: Dez. 06, 2020 


\begin{abstract}
Objective: to evaluate the effectiveness of low-frequency ultrasound associated with copaiba and tea tree oil gel in healing skin lesions. Method: quasi-experimental study with pre and post-tests in a single group, carried out in a public hospital in Ceará, Brazil, from March to September 2017, with 14 participants who received 10 treatment sessions. Results: there was an average reduction of more than $20 \mathrm{~cm}^{2}$ from the 1st to the 5th application. The reduction in the initial size for the 10 th session was over 50\%. There was statistical significance $(p=0.0043)$ between the initial evaluation and the 10th therapy evaluation, with an average reduction of $37 \mathrm{~cm}^{2}$. There was a reduction in the intensity of pain reported by the participants and $62.5 \%$ were free of pain after applying the therapy. Conclusion: the use of the gel with copaiba and tea tree oil points to an alternative coverage associated with biostimulators such as ultrasound. The composition of the gel oil helped in the application and in the reduction of the maceration of the edge of the wounds, as well as the control of the exudate of the lesion.
\end{abstract}

DESCRIPTORS: Healing; Ultrasound therapy; Volatile oils. Stomatherapy.

\title{
RESUMEN
}

Objetivo: Evaluar la efectividad de la ecografía de baja frecuencia asociada con la copaiba y el gel de aceite de árbol de té en la curación de lesiones cutáneas. Método: Estudio cuasiexperimental con pre y pospruebas en un solo grupo, realizado en un hospital público de Ceará, Brasil, de marzo a septiembre de 2017, con 14 participantes que recibieron 10 sesiones de tratamiento. Resultados: Hubo una reducción promedio de más de $20 \mathrm{~cm}^{2}$ de la primera a la quinta aplicación. La reducción del tamaño inicial para la décima sesión fue superior al 50\%. Hubo significación estadística ( $\mathrm{p}=0,0043$ ) entre la evaluación inicial y la décima evaluación de la terapia, con una reducción promedio de $37 \mathrm{~cm}^{2}$. Hubo una reducción en la intensidad del dolor informada por los participantes y el 62,5\% no sintió dolor después de aplicar la terapia. Conclusión: El uso del gel con copaiba y aceite de árbol de té apunta a una cobertura alternativa asociada a los bioestimuladores, como la ecografía. La composición del aceite en gel ayudó en la aplicación y en la reducción de la maceración del borde de las heridas, así como en el control del exudado de la lesión.

DESCRIPTORES: Cicatrización de heridas; Terapia de ultrasonido; Aceites volátiles.

\section{INTRODUÇÃO}

$\mathrm{O}$ uso de recursos fisioterapêuticos pode desencadear efeitos positivos no processo de cicatrização de lesões de pele, que aceleram as diferentes fases do reparo tecidual e reduzem o tempo de lesão por meio da aplicação de recursos eletrofísicos ${ }^{1}$.

Entre os recursos fisioterapêuticos utilizados para acelerar o processo de reparo tecidual está o ultrassom de baixa frequência (USBF). As ondas de ultrassom foram recentemente desenvolvidas para o tratamento de diferentes feridas de difícil cicatrização, com resultados terapêuticos promissores ${ }^{2}$.

O USBF caracteriza-se como uma onda mecânica em que sua energia vibracional é decomposta em energia molecular, o que permeia múltiplos efeitos terapêuticos, como aumento da vascularização e síntese de colágeno, além de minimizar o processo inflamatório, o que impulsiona à reparação dos tecidos ${ }^{3}$.

Os avanços alcançados na área de cuidados com feridas também se apropriou do conhecimento e cultura popular, como os óleos essenciais (OEs). Os OEs são metabólitos secundários extraídos de variadas partes de plantas, que apresentam hidrocarbonetos terpenos, com predomínio de monoterpenos, álcoois e sesquiterpenos, que possuem características bactericidas que ajudam a cicatrização $0^{4,5}$.

Óleos de copaíba (Copaifera sp.) e melaleuca (Melaleuca alternifólia) têm mostrado a sua eficácia no tratamento de feridas e no processo de cicatrização por conter atividade antibacteriana, anti-inflamatória, antiviral, antifúngica e analgésica ${ }^{6,7}$.

A literatura infere êxito na associação de coberturas tópicas e USBF para tratamento de lesões de pele. O USBF propicia a fonoforese e, assim, as substâncias que se encontram sobre a lesão recebem ajuda das ondas geradas para penetrar com maior facilidade nas camadas da pele, acelerando sua absorção e seus efeitos sobre a lesão ${ }^{8}$.

O estudo torna-se relevante por buscar identificar os efeitos de recurso fisioterapêutico utilizado no tratamento de feridas em associação com OEs de copaíba e melaleuca em forma de gel, com base na regeneração do tecido lesado que poderá contribuir com a melhora na qualidade de vida de pessoas com lesões de pele e minimizar o risco de amputações não traumáticas de membros inferiores.

O objetivo do estudo é avaliar a eficácia do USBF associado ao gel com óleos de copaíba e melaleuca na cicatrização de lesões de pele. 


\section{MÉTODO}

\section{Desenho do estudo}

Estudo quase-experimental com grupo único de pré e pós-teste, realizado em hospital terciário no interior do estado do Ceará, Brasil, no período de março a setembro de 2017.

\section{Seleção de participantes}

Os participantes foram recrutados em ambulatório de estomaterapia e clínicas de internação médica e cirúrgica do referido hospital. Foram incluídos no estudo indivíduos maiores de 18 anos; que apresentassem lesões com perda de epiderme, derme e/ou tecido subcutâneo com necessidade de acompanhamento ou ferimentos crônicos; e ser acompanhado no hospital, no setor da internação ou ambulatório.

Foram excluídos pacientes com incapacidade de comparecer ao hospital; apresentação de três ou mais fatores que prejudicam a cicatrização: diabetes mellitus, tabagismo, doença autoimune, uso de corticoides, tecido desvitalizado com necrose, indicação de amputação do membro ou hipersensibilidade na ferida. A exclusão de pacientes com três ou mais fatores que prejudicam a cicatrização se deve ao fato de que isso poderia gerar viés na efetividade da terapêutica com USBF, visto que esses pacientes já teriam tendência a um processo de cicatrização mais demorado.

Dos 35 participantes recrutados, 14 possuíram disponibilidade para concluir a intervenção (mínimo de 10 sessões com a terapia ultrassônica associada ao gel com óleo de copaíba e melaleuca), os quais compuseram a amostra da pesquisa.

\section{Coleta de dados}

A coleta de dados foi efetivada por meio da aplicação de um formulário, estruturado em três partes: 1) questões referentes ao perfil sociodemográfico (idade, sexo, escolaridade, saneamento básico); 2) questões relacionadas aos aspectos clínicos dos participantes (hipertensão arterial, diabetes mellitus tipo 2 e dias de acompanhamento da ferida); 3) dados de identificação das lesões (tipo de pele, tratamento utilizado, tipo de ulceração, aparência, deslocamento e aspectos do bordo da ferida).

Para mensurar as lesões foi utilizada a Pressure Ulcer Scale for Healing (PUSH), que compila dados referentes à área da lesão, tipo de tecido e quantidade de exsudato, gerando um valor em escore que pode ser inserido em um gráfico e acompanhar a evolução da cicatrização da lesão.
Houve avaliação da dor antes, durante e logo após a intervenção, através da Escala Visual Analógica (EVA), em cada sessão de tratamento. A dor também foi mensurada por meio de um inquérito sobre a dor, sendo classificada em: 1 - dor aguda não-cíclica; 2 - dor aguda cíclica; 3 - dor crônica; e 4 - sem dor. Foi considerada a dor aguda cíclica como a dor intermitente que cessa e recomeça em períodos do dia por menos de seis meses; dor aguda não cíclica como a dor que não cessa em período menor que seis meses; e dor crônica como a dor por período maior que seis meses. A utilização do ultrassom foi registrada quanto ao dia da aplicação, código referente à lesão e tempo de aplicação.

\section{Processo de intervenção}

O ultrassom utilizado foi o Sonopulse III Ibramed - $1 \mathrm{Mhz}$ e $3 \mathrm{Mhz}$, com 21W de potência e diâmetro da área de irradiação efetiva (ERA) de 3,5cm. A aplicação do USBF nas lesões foi realizada por três enfermeiros.

Primeiramente, realizou-se a limpeza da lesão com soro fisiológico a 0,9\%. Em seguida, aplicou-se o gel com óleos de copaíba e melaleuca no leito da ferida, respeitando os bordos da lesão, com camada de aproximadamente $0,3 \mathrm{~mm}$, seguido da aplicação de filme de policloreto de polivinila (PVC) em volta da lesão para manter a margem de segurança das bordas. Logo após, envolveu-se com PVC o transdutor do ultrassom e a foi realizada a aplicação de agente de impedância acústica em forma de um gel a base de água.

Após esse primeiro momento, houve a aplicação do transdutor com início nos bordos da lesão com extensão ao centro da ferida, tendo a margem de $1 \mathrm{~cm}$ entre as áreas de aplicação. As lesões foram medidas com uma fita métrica em centímetros quadrados na primeira, quinta e décima aplicação do ultrassom.

Os participantes foram acompanhados em dias alternados e a técnica da aplicação do ultrassom seguiu padronização de variância de acordo com o tamanho e característica da lesão, na modalidade pulsada, densidade de energia de $3 \mathrm{~W} / \mathrm{cm}^{2}$ e frequência de $1 \mathrm{MHz}$. O ultrassom foi aplicado em movimentos em forma de oito, suavemente, em sentido anti-horário ${ }^{2}$.

O tempo estimado da aplicação foi determinado pelo cálculo do comprimento multiplicado pela largura da lesão e dividido pela ERA. Após cada cálculo individual, colocava-se no aparelho o tempo de aplicação, medido em minutos. Ao finalizar o tempo calculado, o USBF disparava o alarme para sinalizar o fim da intervenção. 


\section{Análise dos dados}

Os dados coletados foram tabulados no programa Excel 2016 e analisados no programa estatístico Statistical Package for the Social Sciences (SPSS), versão 20. Os dados categóricos foram apresentados em frequência absoluta e percentual, e comparados através dos testes exato de Fisher ou Quiquadrado de Pearson. Foi realizado teste de normalidade de Kolmogorov-Smirnov nos dados quantitativos, os quais foram expressos em forma de média e erro-padrão da média, com análise por meio do teste de Friedman, em seguida, pós-teste de Dunn e correlação de Spearman. O Teste t de Student foi utilizado para comparação das médias das áreas das lesões. Adotou-se nível de significância de 5\%.

\section{Aspectos éticos}

Esta pesquisa recebeu aprovação do Comitê de Ética em Pesquisa (CEP) da Universidade Internacional da Integração da Lusofonia Afro-Brasileira, mediante parecer $\mathrm{n}^{\circ} 1.049 .373 / 2015$. Todas as consignações da Resolução n. ${ }^{\circ}$ 466/12 do Conselho Nacional de Saúde foram seguidas e todos os participantes assinaram o Termo de Consentimento Livre e Esclarecido.

\section{RESULTADOS}

Houve participação de 14 pacientes, que somaram 17 lesões. Os participantes tinham como principais características a predominância do sexo masculino $(71,4 \%)$, com idade média de 59,9 anos $( \pm 18,7)$, aposentados $(54,1 \%)$ e agricultores $(28,5 \%)$, autorreferidos como pardos $(85,7 \%)$ e analfabetos $(71,4 \%)$. O diagnóstico de diabetes mellitus tipo 2 esteve presente em 5 participantes, que realizavam tratamento da doença (35,7\%). Entre os pacientes hipertensos $(n=5)$, somente um realizava tratamento medicamentoso da doença. Apenas um participante afirmou ser etilista.

A Tabela 1 apresenta informações referentes às lesões e a pele dos participantes submetidos ao USBF. Houve predomínio de pele muito fina $(29,4 \%)$ e descorada (23,5\%). Em relação ao tipo de ferida, pé diabético $(35,3 \%)$ e lesões por pressão $(29,4 \%)$ foram prevalentes, com bordo que apresentava eritema $(47,1 \%)$. No leito da lesão o esfacelo esteve presente na maioria das lesões na avaliação inicial $(58,8 \%)$. A dor (aguda cíclica e não cíclica) também esteve presente na maioria das lesões.
Tabela 1. Distribuição das características das lesões e da pele dos pacientes em uso da irradiação ultrassônica de baixa frequência associada ao gel com copaíba e melaleuca. Acarape (CE), Brasil - 2017.

\begin{tabular}{|c|c|}
\hline Tipo pele - início da aplicação & $\mathrm{n}(\%)$ \\
\hline Muito fina & $05(29,4)$ \\
\hline Seca & $03(17,6)$ \\
\hline Descorada & $04(23,5)$ \\
\hline Úmida & $02(11,8)$ \\
\hline Com edema & $03(17,6)$ \\
\hline Tipo da ferida & n (\%) \\
\hline Úlcera venosa & $02(11,8)$ \\
\hline Úlcera arterial & $01(5,9)$ \\
\hline Lesão por pressão & $05(29,4)$ \\
\hline Pé diabético & $06(35,3)$ \\
\hline Traumática & $02(11,8)$ \\
\hline Outra & $01(5,9)$ \\
\hline Aparência & $\mathrm{n}(\%)$ \\
\hline Com necrose & $04(23,5)$ \\
\hline Com infecção & $03(17,6)$ \\
\hline Com esfacelo & $10(58,8)$ \\
\hline Tratamento tópico utilizado & $\mathrm{n}(\%)$ \\
\hline Nenhum & $01(5,9)$ \\
\hline Papaína & $02(11,8)$ \\
\hline Alginato & $03(17,6)$ \\
\hline Ácidos Graxos Essenciais & $06(35,3)$ \\
\hline Colagenase & $04(23,5)$ \\
\hline Outros & $01(5,9)$ \\
\hline Dor & $\mathrm{n}(\%)$ \\
\hline Não & $05(29,4)$ \\
\hline Dor aguda não cíclica & $05(29,4)$ \\
\hline Dor aguda cíclica & $05(29,4)$ \\
\hline Dor crônica & $02(11,8)$ \\
\hline Deslocamento do bordo & $\mathrm{n}(\%)$ \\
\hline Não & $14(82,4)$ \\
\hline $\mathrm{Sim}$ & $03(17,6)$ \\
\hline Bordo & $\mathrm{n}(\%)$ \\
\hline Eritema e calor & $08(47,1)$ \\
\hline Borda endurecida & $03(17,6)$ \\
\hline Rompimento & $03(17,6)$ \\
\hline Pele descamada e seca ao redor da ferida & $03(17,6)$ \\
\hline
\end{tabular}

A avaliação das aplicações por meio da média da área da lesão revelou redução média superior a $20 \mathrm{~cm}^{2}$, da $1^{\text {a }}$ para a $5^{\mathrm{a}}$ aplicação. A redução do tamanho inicial até a $10^{\mathrm{a}}$ sessão foi superior a $50 \%$. $\mathrm{Na}$ avaliação, houve lesões que apresentaram cicatrização na $10^{a}$ sessão $(n=2)$. Salienta-se a variação das áreas das lesões, onde valores mínimos e máximos encontravam-se distantes (Tabela 2). 
Tabela 2. Distribuição das aplicações do ultrassom de baixa frequência e a média da área das lesões avaliadas antes da aplicação inicial, após cinco aplicações e dez aplicações. Acarape (CE), Brasil - 2017.

\begin{tabular}{|c|c|c|c|c|c|}
\hline $\mathrm{N}^{\circ}$ de aplicações de ultrassom & $\mathrm{N}$ & Média da área $\left(\mathrm{cm}^{2}\right)$ & Desvio Padrão \pm & Min & Max \\
\hline 0 aplicações & 17 & 64.18 & 79.66 & 5 & 270 \\
\hline 5 Aplicações & 17 & 43.65 & 53.99 & 4 & 182 \\
\hline 10 Aplicações & 17 & 27.18 & 42.87 & 0 & 156 \\
\hline
\end{tabular}

A Tabela 3 aponta diferenças entre as médias das áreas das lesões nos três momentos de avaliação. Houve maior significância estatística $(\mathrm{p}=0,0043)$ entre a avaliação inicial e décima aplicação do USBF associado ao gel com copaíba e melaleuca, com redução média de $37 \mathrm{~cm}^{2}$.

Tabela 3. Diferença de médias para duas amostras pareadas entre os períodos de aplicação do ultrassom de baixa frequência associado ao gel com copaíba e melaleuca. Acarape (CE), Brasil - 2017.

\begin{tabular}{|c|c|c|c|c|c|}
\hline $\mathrm{N}^{\circ}$ de aplicações de ultrassom & $\mathrm{N}$ & Média $\left(\mathrm{cm}^{2}\right)$ & Erro Padrão & $\mathrm{T}$ & $P$ \\
\hline 0 aplicações & 17 & 64.176 & 19.320 & & \\
\hline 5 aplicações & 17 & 43.647 & 13.095 & 2.899 & 0,011 \\
\hline Diferença & & $20.529^{\star \star}$ & 23.340 & & \\
\hline 0 aplicações & 17 & 64.176 & 19.320 & & \\
\hline 10 aplicações & 17 & 27.176 & 10.397 & 3.323 & 0,0043 \\
\hline Diferença & 17 & $37.000^{* \star *}$ & 11.135 & & \\
\hline 5 aplicações & 17 & 43.647 & 13.095 & & \\
\hline 10 aplicações & 17 & 27.176 & 10.397 & 3.069 & 0,007 \\
\hline Diferença & 17 & $16.471^{* * *}$ & 5.367 & & \\
\hline
\end{tabular}

$\mathrm{T}=$ Teste $\mathrm{T}$ de Student; $\mathrm{P}=\mathrm{P}$-valor; **Significativo a 5\%; ${ }^{* * *}$ Significativo a $1 \%$.

A Fig. 1 apresenta a distribuição das médias, com diferença estatística na redução da área entre a média inicial e a $5^{\text {a }}$ aplicação, e entre a média na $5^{\mathrm{a}}$ avaliação para a $10^{\mathrm{a}}$ aplicação do USBF associado ao gel com copaiba e melaleuca $(\mathrm{p}<0,005)$.

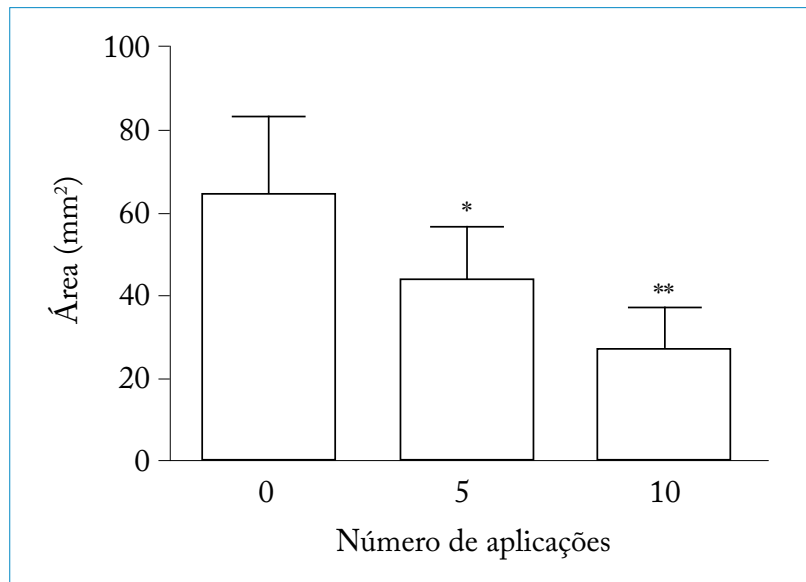

Figura 1. Distribuição das médias entre a média inicial, quinta aplicação e décima aplicação do ultrassom de baixa frequência associado ao gel com copaíba e melaleuca. Acarape (CE), Brasil - 2017.

${ }^{*} p<0,05$ versus 0 aplicações; * $p<0,05$ versus 5 aplicações, teste de Friedman/Dunn (média \pm EPM).
Na Fig. 2 observa-se a regressão logística para identificar o tempo para cicatrização da lesão com a aplicação do USBF associado ao gel com copaiba e melaleuca. Foi utilizado o modelo de regressão entre o número de aplicações e o tamanho médio da lesão.Identificou-se a necessidade de 17 aplicações para estimar área média de lesão de $0 \mathrm{~cm}^{2}\left(\mathrm{p}=0,026, \mathrm{r}=-0,311, \mathrm{r}^{2}=0,061\right)$. $\mathrm{Na}$ correlação de Spearman foi identificado valor de $\mathrm{p}<0,05$.

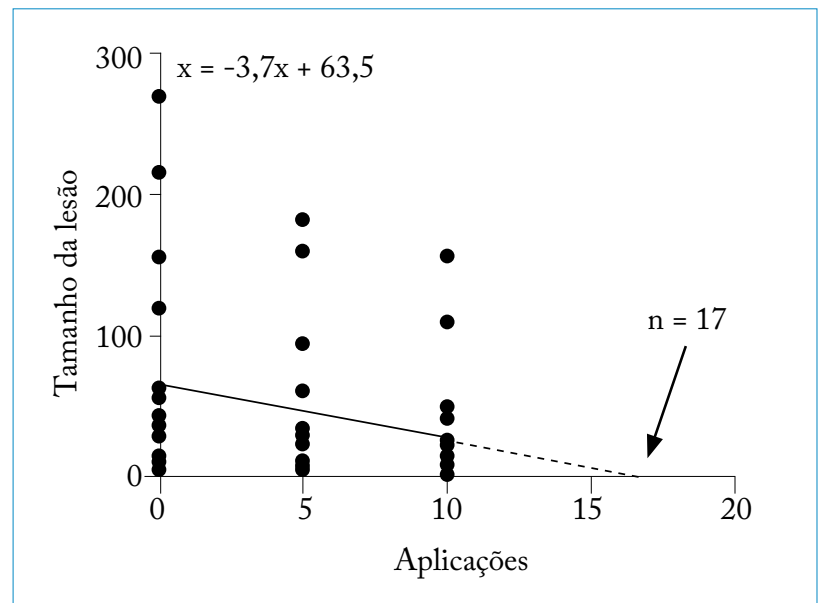

Figura 2. Regressão logística do número de aplicações e a média das lesões. Acarape (CE), Brasil - 2017. 
A Tabela 4 apresenta a intensidade da dor referida pelos pacientes em quatro níveis: intensa, moderada, leve e sem dor. Sendo avaliados em três cortes de tempo: antes, durante e após a aplicação da terapêutica com ultrassom. Houve redução da intensidade da dor referida pelos pacientes e $62,5 \%$ estavam isentos de dor após a aplicação da terapêutica.
$\mathrm{Na}$ Fig. 3, a avaliação da PUSH revela redução da área em todas as lesões avaliadas.

Quatro lesões não apresentaram redução da pontuação entre a $5^{\mathrm{a}}$ e $10^{\mathrm{a}}$ aplicação do USBF (lesões 06, 08, 10 e 17). Duas lesões apresentaram cicatrização total (Fig. 3).

Tabela 4. Distribuição da avaliação da dor antes, durante e após as aplicações do ultrassom de baixa frequência associado ao gel com copaíba e melaleuca. Acarape (CE), Brasil - 2017.

\begin{tabular}{|c|c|c|c|c|}
\hline Dor & Antes & Durante & Após & $p^{*}$ \\
\hline Intensa & $5(31,3 \%)$ & $2(12,5 \%)$ & $1(6,3 \%)$ & \multirow{4}{*}{0,227} \\
\hline Moderada & $4(25,0 \%)$ & $4(25,0 \%)$ & $3(18,8 \%)$ & \\
\hline Leve & $4(25,0 \%)$ & $2(12,5 \%)$ & $2(12,5 \%)$ & \\
\hline Sem dor & $3(18,8 \%)$ & $8(50,0 \%)$ & $10(62,5 \%)$ & \\
\hline
\end{tabular}

* Teste qui-quadrado de Pearson.

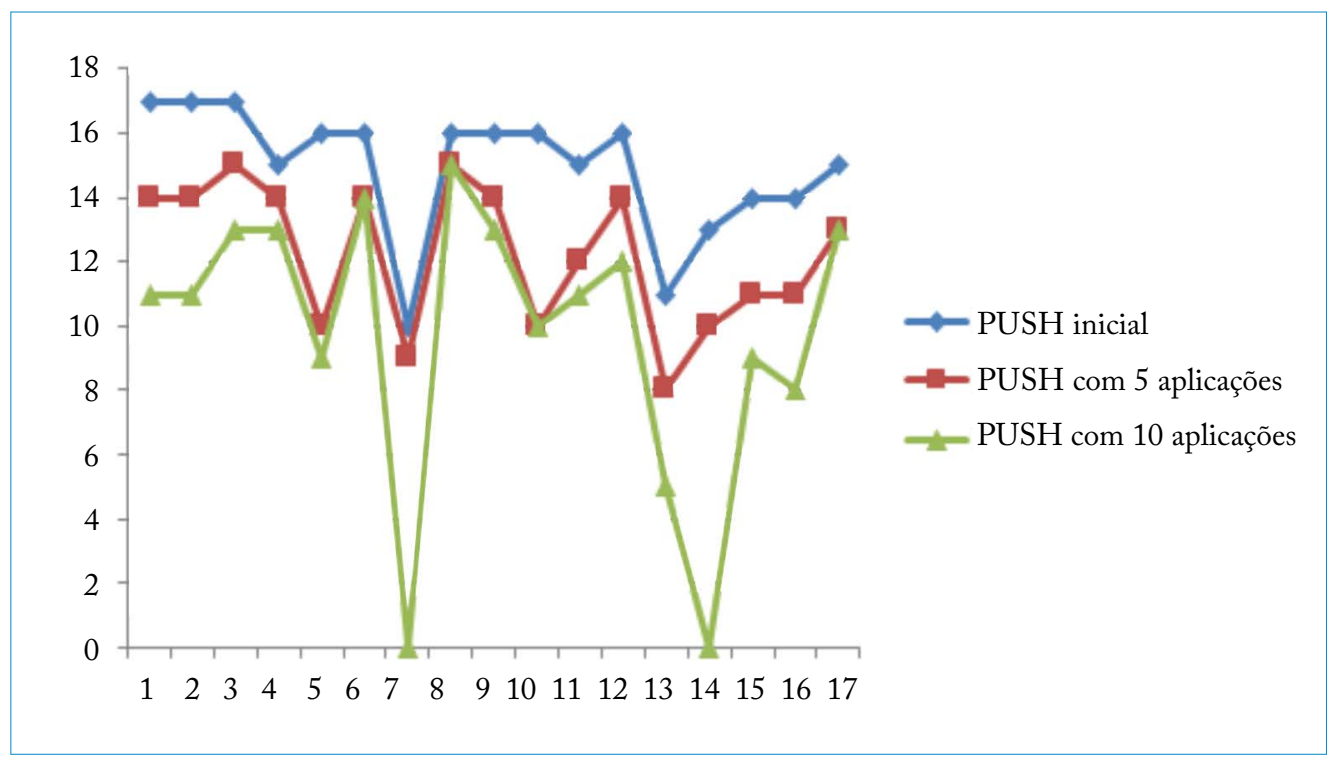

Figura 3. Distribuição da pontuação da Pressure Ulcer Scale for Healing na avaliação inicial, após cinco e dez aplicações. Acarape (CE), Brasil - 2017.

\section{DISCUSSÃO}

As características das lesões e seu tipo convieram para identificar fatores que podem dificultar a cicatrização de feridas, principalmente relacionado ao tipo de pele e bordo da lesão.

Em relação à classificação das feridas, a maioria caracteriza-se como pé diabético seguida de lesão por pressão. Achado discrepante ao panorama epidemiológico da região do estudo, em que as úlceras venosas e lesão por pressão são predominantes ${ }^{9,10}$. Tais lesões crônicas apresentam dificuldades de cicatrização e requerem que novas terapias adjuvantes sejam utilizadas em associação com o tratamento convencional. Pesquisas com terapias adjuvantes como o USBF estão em avanço constante e seus efeitos vêm sendo descritos ao longo dos tempos e da prática clínica ${ }^{11}$.

Estudo consultado, que associou USBF com vitaminas em lesões tegumentares, identificou que o tratamento foi mais eficaz na primeira fase do processo de cicatrização, com maior maturação das fibras colágenas ${ }^{8}$.

As ondas de baixa frequência podem acelerar a velocidade de cicatrização de feridas abertas bem como lesões nos tecidos profundos ${ }^{2}$. Assim, a possibilidade de 
estímulos em tecidos profundos aponta o USBF como uma ferramenta que acelera o processo de cicatrização e previne novas lesões, contudo, as pesquisas ainda não mostram um protocolo específico para o tratamento das diferentes lesões ${ }^{12}$.

Dessa maneira, o número de aplicações da terapia ultrassônica estabelecida no estudo foi de dez aplicações, por ser perceptível que a utilização da radiação de baixa intensidade perde eficácia conforme o número de sessões subsecutivas ${ }^{13}$.

A disparidade de aplicações e parâmetros utilizados pode culminar no uso empírico do USBF por alguns profissionais, gerando divergência nos resultados encontrados em diferentes pesquisas ${ }^{14}$.

Neste estudo, o gel com OEs de copaíba e melaleuca foi utilizado em associação com a terapia ultrassônica. Em outros estudos ${ }^{15,16}$, o óleo de copaíba apresentou transmissibilidade positiva para utilização por fonoforese do USBF, atividade antimicrobiana, o que cessou a exsudação purulenta na área da lesão e contribuiu, ainda, para a multiplicação e avanço do tecido de granulação, e o óleo de melaleuca inibiu o crescimento de fungos e bactérias ${ }^{17}$.

A análise da distribuição das médias das lesões revelou diferença estatística na redução da área entre a média inicial e a $5^{\mathrm{a}}$ aplicação, e entre a média na $5^{\mathrm{a}}$ avaliação para a $10^{a}$ aplicação do USBF $(p<0,005)$. Esse achado mostra-se relevante por indicar ação benéfica no progresso da cicatrização de lesões de variadas etiologias.

Estudos na Europa identificaram, respectivamente, maior redução média da área da ferida no grupo de tratamento com USBF (67,0\%), além de diminuir o exsudato e dor do paciente, dispersar biofilmes e aumentar a cicatrização em feridas de várias etiologias ${ }^{18,19}$. O princípio do USBF também ativa os fibroblastos nos tecidos moles, o que ajuda no progresso da regeneração dos tecidos e a síntese de proteínas por meio de um processo de cavitação e micro-fluxo ${ }^{20}$.

A redução observada na área das lesões, mesmo em indivíduos com doenças de base, como hipertensão e diabetes que dificultam a circulação vascular e consequentemente a oxigenação, pode ser justificada pelo fato da terapia ultrassônica de baixa frequência elevar o metabolismo, proliferação celular e oxigenação local.

Em estudo asiático, resultados evidenciaram que a aplicação do USBF em feridas por diabetes promove a expressão do fator de crescimento endotelial, fator de crescimento transformador, inibe a expressão de interleucina e fator de necrose tumoral ${ }^{21}$.

A USBF apresenta aspectos positivos ao processo de cicatrização, desde a redução da área da lesão até o aumento do tecido viável, o que estimula a cicatrização mais rápida em comparação com técnicas convencionais de curativos, com um tecido cicatricial mais resistente ${ }^{11}$.

Além disso, neste estudo, os pacientes referiram melhora da dor durante e após a aplicação do USBF, reafirmando assim o efeito analgésico da terapia ultrassônica, o que corrobora outro estudo consultado, em que todos os pacientes apresentaram redução significativa da dor após tratamento com $U_{S B F^{19}}$. Esse é um dado importante, uma vez que a dor é um dos sintomas mais referidos por indivíduos com feridas. A dor afeta tanto a área da lesão quanto as áreas adjacentes, podendo afetar até mesmo o membro inteiro ${ }^{22}$.

Vale ressaltar que o USBF não possui, até o momento, relatos de eventos adversos gerados em decorrência da sua aplicação. Assim, ele possui aplicabilidade terapêutica para analgesia, sendo uma fonte de alívio da dor a ser considerada no ambiente hospitalar/ambulatorial.

O USBF é um recurso adjuvante que pode ser utilizado na cicatrização de feridas. Essa tecnologia vem sendo utilizada há alguns anos no cuidado de feridas, principalmente por profissionais da área de fisioterapia, no entanto a enfermagem vem se apropriando do uso desse recurso, o que infere a necessidade de novas publicações na área da enfermagem para que haja maior compreensão e utilização dessa tecnologia adjuvante no tratamento de lesões de pele.

A associação do USBF com outros agentes cicatrizantes no leito da ferida deve ser mais bem investigada para potencializar e divulgar seus efeitos. O tempo de aplicação do ultrassom foi um fator limitante, tendo em vista lesões com grandes áreas para aplicação, o que pode limitar seu uso na rotina hospitalar e/ou ambulatorial.

\section{CONCLUSÃO}

O uso do gel com óleo de copaíba e melaleuca aponta para uma alternativa de cobertura associada a bioestimuladores como o USBF, pelo efeito de fonoforese. 
A composição do óleo em gel auxiliou na aplicação e na redução de maceração do bordo das feridas bem como o controle do exsudato da lesão. A intervenção com USBF associado ao gel com óleo de copaíba e melaleuca foi realizada sem intercorrências e se apresentou como um recurso terapêutico para a redução da área de feridas bem como sua cicatrização.

\section{CONTRIBUIÇÃO DOS AUTORES}

\author{
Conceitualização: Mororó DGA; Brandão MGSA e
} Ponte VA; Investigação: Mororó DGA; Ponte VA; Silva ASJ e Batista FWS; Redação - Primeira versão: Mororó DGA; Brandão MGSA; Ponte VA; Veras VS e Araújo TM; Redação - Revisão \& Edição: Brandão MGSA e Araújo TM.

\section{REFERÊNCIAS}

1. Furtado RAA, Noleto MLP, Pessoa DR, Almeida VS, Filho ALMM, Uchôa VT et al. Ação do gel Anacardium Occidentale L. associado ao ultrassom terapêutico no processo de cicatrização em camundongos. Saúde (Sta Maria) 2019;45(2):1-15. https://doi.org/10.5902/2236583435474

2. Alkahtani SA, Kunwar PS, Jalilifar M, Rashidi S, Yadollahpour A. Ultrasound-based Techniques as Alternative Treatments for Chronic Wounds: A Comprehensive Review of Clinical Applications. Cureus 2017;9(12):e1952. https://doi. org/10.7759/cureus.1952

3. Bagnato VS, Paolillo AR, João JP, Paolillo FR, João HA, Frascá D. Dois em um: dispositivo usa ultrassom e laser simultaneamente para reabilitar pacientes com artrose [Depoimento a Yuri Vasconcelos]. Pesquisa FAPESP 2015;(229):76-77. Disponível em: https://revistapesquisa. fapesp.br/2015/03/13/dois-em-um/

4. Nepomoceno TAR, Pietrobon AJ. Melaleuca alternifolia: uma revisão sistemática da literatura brasileira. Rev UNINGÁ Review 2020;35(eRUR3409):1-29. Disponível em: http://revista.uninga.br/index.php/uningareviews/article/ view/3409/2207

5. Silva LL, Almeida R, Verícimo MA, Macedo HW, Castro HC. Atividades terapêuticas do óleo essencial de melaleuca (melaleuca alternifolia): Uma revisão de literatura. Braz J Hea Rev 2019;2(6):6011-21. https://doi.org/10.34119/ bjhrv2n6-094

6. Cavalari TGF, Oliveira ACC. Óleo essencial de melaleuca. Saúde Foco 2017;9:580-6. Disponível em: https:// portal.unisepe.com.br/unifia/wp-content/uploads/ sites/10001/2018/06/065_essencialdemelaleuca.pdf

7. Pires VGA, Moura MR. Preparação de novos filmes poliméricos contendo nanoemulsões do óleo de melaleuca, copaíba e limão para aplicação como biomaterial. Quim Nova 2017;40(1):1-5. https://doi.org/10.21577/01004042.20160130

8. Guimarães NG, Pires-De-Campos MSM, Leonardi GR, Dib-Giusti HHK, Polacow MLO. Efeito do ultrassom e do dexapantenol na organização das fibras colágenas em lesão tegumentar. Rev Bras Fisioter 2011;15(3):227-32. Disponível em: http://www.scielo.br/pdf/rbfis/v15n3/09.pdf
9. Lentsck MH, Baratieri T, Trincaus MR, Mattei AP, Miyahara CTS. Quality of life related to clinical aspects in people with chronic wound. Rev Esc Enferm USP 2018;52:e03384. https://doi.org/10.1590/S1980-220X2017004003384

10. Barros MPL, Ferreira PJO, Maniva SJC, Holanda RE. Caracterização de feridas crônicas de um grupo de pacientes acompanhados no domicílio. R Interd 2016;9(3):111. Disponível em: https://dialnet.unirioja.es/servlet/ articulo?codigo $=6772005$

11. Ponte VA, Silva ASJ, Mororó DGA, Veras VS, Araújo TM. Avaliação dos Efeitos da Irradiação Ultrassônica de Baixa Frequência no Tratamento de Úlcera Venosa. Rev Fund Care Online 2019;11(5):1219-25. https://doi.org/10.9789/21755361.2019.v11i5.1219-1225

12. Jalilifar M, Ali Y, Zohre R, Samaneh R. Electromagnetic fields and ultrasound waves in wound treatment: a comparative review of therapeutic outcomes. Biosci Biotech Res Asia 2015; 12 (spl1):185-95. https://doi.org/10.13005/bbra/1622

13. Wang R, Feng $Y$, Di B. Comparisons of negative pressure wound therapy and ultrasonic debridement for diabetic foot ulcers: a network meta-analysis. Int J Clin Exp Med 2015;8(8):12548-56. Disponível em: https://www.ncbi.nlm. nih.gov/pmc/articles/PMC4612850/pdf/ijcem0008-12548.pdf

14. Korelo RIG, Fernandes LC. Ultrassom terapêutico para cicatrização de feridas: revisão sistemática. ConScientiae Saúde 2016;15(3):518-29. https://doi.org/10.5585/ conssaude.v15n3.6421

15. Marinho DF, Oliveira ECP, Araújo JAS, Pinto IF, Lima HS, Moraes WP et al. Avaliação da transmissibilidade ultrassônica do gel fitoterápico de Copaifera duckei Dwyer. Pesq Vet Bras 2017;37(5):516-20. https://doi.org/10.1590/ s0100-736×2017000500015

16. Martins IFB, Silva A. Influência do óleo de copaíba (copaifera sp.) no tratamento de ferida cutânea infeccionada. R pesq cuid fundam online 2010;2(Ed. Supl.):526-9. Disponível em: https://www.redalyc.org/pdf/5057/505750987035.pdf

17. Tobouti PL, Mussi MCM, Rossi DCP, Pigatti FM, Taborda CP, Taveira LAA et al. Influence of melaleuca and copaiba oils on Candida albicans adhesion. Gerodontology 2014;33(3),3805. https://doi.org/10.1111/ger.12172 
18. Wiegand C, Bittenger K, Galiano RD, Driver VR, Gibbons GW. Does non-contact low-frequency ultrasound (NLFU) therapy contribute to wound healing at the molecular level? Wound Repair Regen 2017;25(5):871-82. https://doi.org/10.1111/ wrr.12595

19. Chang YR, Perry J, Cross K. Low-Frequency Ultrasound Debridement in Chronic Wound Healing: A Systematic Review of Current Evidence. Plast Surg (Oakv) 2017;25(1):216. https://doi.org/10.1177/2292550317693813

20. Lee WS, Park ES, Kang SG, Tak MS, Kim CH. Successful Treatment of Recalcitrant Remaining Postoperative Wounds by Dual-Frequency Ultrasound. Med Laser 2018;7(2):74-8. https://doi.org/10.25289/ML.2018.7.2.74

21. Chen L, Zheng Q, Chen X, Wang J, Wang L. Low frequency ultrasound enhances vascular endothelial growth factor expression, thereby promoting the wound healing in diabetic rats. Exp Ther Med 2019;18(5):4040-8. https://doi. org/10.3892/etm.2019.8051

22. Nascimento LA, Cardoso MG, Oliveira SA, Quina E, Sardinha DSS. Manuseio da dor: avaliação das práticas utilizadas por profissionais assistenciais de hospital público secundário. Rev Dor 2016;17(2):76-80. https://doi.org/10.5935/18060013.20160019 